\title{
Macroscopic and microscopic spectral properties of brain networks during local and global synchronization
}

\author{
Vladimir A. Maksimenko, ${ }^{1}$ Annika Lüttjohann, ${ }^{2}$ Vladimir V. Makarov, ${ }^{1}$ Mikhail V. Goremyko, ${ }^{1}$ Alexey A. Koronovskii, ${ }^{3}$ \\ Vladimir Nedaivozov, ${ }^{1}$ Anastasia E. Runnova, ${ }^{1}$ Gilles van Luijtelaar, ${ }^{4}$ Alexander E. Hramov, ${ }^{1}$ and Stefano Boccaletti ${ }^{5,6}$ \\ ${ }^{1}$ Yuri Gagarin State Technical University of Saratov, REC “Nonlinear Dynamics of Complex Systems," Saratov 410054, Russia \\ ${ }^{2}$ University of Münster, Institute of Physiology I, Münster 48149, Germany \\ ${ }^{3}$ Saratov State University, Faculty of Nonlinear Processes, Saratov 410012, Russia \\ ${ }^{4}$ Radboud University, Donders Centre for Cognition, Nijmegen 6525 HR, Netherlands \\ ${ }^{5}$ CNR-Institute for complex systems, Sesto Fiorentino 50019, Italy \\ ${ }^{6}$ The Italian Embassy in Tel Aviv, Tel Aviv 68125, Israel \\ (Received 21 January 2017; revised manuscript received 29 April 2017; published 20 July 2017)
}

\begin{abstract}
We introduce a practical and computationally not demanding technique for inferring interactions at various microscopic levels between the units of a network from the measurements and the processing of macroscopic signals. Starting from a network model of Kuramoto phase oscillators, which evolve adaptively according to homophilic and homeostatic adaptive principles, we give evidence that the increase of synchronization within groups of nodes (and the corresponding formation of synchronous clusters) causes also the defragmentation of the wavelet energy spectrum of the macroscopic signal. Our methodology is then applied to getting a glance into the microscopic interactions occurring in a neurophysiological system, namely, in the thalamocortical neural network of an epileptic brain of a rat, where the group electrical activity is registered by means of multichannel EEG. We demonstrate that it is possible to infer the degree of interaction between the interconnected regions of the brain during different types of brain activities and to estimate the regions' participation in the generation of the different levels of consciousness.
\end{abstract}

DOI: 10.1103/PhysRevE.96.012316

\section{INTRODUCTION}

The current trends in neuroscience and neurophysiology are connected with the analysis of brain networks [1-4], which interact with each other to perform different types of cognitive tasks, as, e.g., the formation of a memory trace [5,6], the processing of a visual object [7,8], or the development (on a clinical level) of pathological rhythms, like epileptic seizures $[9,10]$. These interactions are often quantified by means of the degree of synchrony, which can be measured both locally (i.e., within the same brain structure) or over a more global scale (i.e., in between brain structures) [11]. While neurophysiology aims at understanding the interplay of individual neurons [12,13], the majority of available data (especially those acquired from human subjects) comes from noninvasive tests. These tests are made, in daily practice, under the form of electroencephalograms (EEG) or magnetoencephalograms (MEG), functional magnetic resonance imaging (fMRI), which actually measure the (electric or magnetic) group activity of large ensembles of cells. The focal riddle for physicists and neuroscientists consists, therefore, in disclosing the way microscopic scale neural interactions pilot the formation of the different activities revealed (at a macroscopic scale) by EEG and MEG signals.

In a network of active elements (like neurons in the brain), one has to distinguish between the signal sensed at a microscopic scale (the individual, electric or magnetic, activity of a neuron) and the macroscopic signal, which is instead produced by a group (or subnetwork) of elements. Processes taking place at the network's microscopic level (such as partial or complete synchronization between units, formation of clusters, etc.) affect the spectral properties of the macroscopic signal.
Starting from a model network of Kuramoto oscillators, which evolve adaptively by means of homophilic and homeostatic adaptive principles, we give evidence that the increase of synchronization within groups of nodes (leading to the formation of structural synchronous clusters) causes also the defragmentation of the wavelet energy spectrum of the macroscopic signal and introduce a practical and computationally not demanding technique that allows an estimation of the interaction between microscopic units of a network by means of appropriate treatment of the macroscopic signals. Our methodology is then applied for getting a glance to the microscopic interactions occurring in a neurophysiological system, namely, in the thalamocortical neural network of an epileptic brain of a rat, where the group electrical activity is registered via multichannel EEG. We compare our technique with wavelet coherence approach and show that both methods provide equivalent results in the analysis of the interaction between the cortex and the thalamus. We demonstrate the possibility to determine the degree of interaction between the interconnected regions of the brain during different types of brain activities.

\section{MODEL NETWORK OF KURAMOTO OSCILLATORS}

The network under consideration is a multiplex [14] graph, in which topology and dynamics of nodes mutually interact via homophily $[15,16]$ and homeostasis [17] principles, as proposed in Refs. $[18,19]$. The units of the network are Kuramoto oscillators [20], the most common and simplest way to describe synchronous phenomena occurring in nature [21-23]. The system consists, therefore, of $M$ layers, each of which are made of $N=300$ oscillators. The phase evolution 
of each oscillator is given by

$$
\begin{aligned}
\dot{\phi}_{i}^{l}(t)= & \omega_{i}+\lambda_{1} \sum_{j \neq i}^{N} w_{i j}^{l}(t) \sin \left(\phi_{j}^{l}-\phi_{i}^{l}\right) \\
& +\lambda_{2} \sum_{j \neq l}^{M} \sin \left(\phi_{i}^{j}-\phi_{i}^{l}\right) .
\end{aligned}
$$

Here, $\phi_{i}^{l}(t)$ is the time-dependent phase of the $i$ th oscillator of the $l$ th layer, dot denotes a temporal derivative, $\left\{\omega_{i}\right\}$ is a set of randomly assigned frequencies [uniformly distributed in the interval $\left.[1,10]\left(\mathrm{s}^{-1}\right)\right]$. Notice that (in the spirit of a multilayer network) the natural frequency $\omega_{i}$ of the $i$ th oscillator is the same in all $M$ layers, whereas its instantaneous phase is actually layer-dependent. $\lambda_{1}$ and $\lambda_{2}$ are the intra- and interlayer coupling strengths, respectively.

At first, all weights $\left\{w_{i j}^{l}(t=0)\right\}$ are randomly assigned in the range $[0,1]$ (except those corresponding to $i=j$, which are all set to zero), and normalized via the condition $\sum_{j \neq i}^{N} w_{i j}^{l}=1(l=1, \ldots, M)$. The latter implies that the input strength received by each unit $i$ in each layer $l$ is constant, as in a homeostatic process. Equation (1) is then initially simulated with fixed weights $\left\{w_{i j}^{l}(t)=w_{i j}^{l}(0)\right\}$, up to $t^{A}=500 \mathrm{~s}$, when the weights start instead to evolve adaptively (so as to enable layers to possibly reorganize), corresponding to a homophily principle, which is expressed by the following equation:

$$
\dot{w}_{i j}^{l}(t)=p_{i j}^{l}(t)-\left[\sum_{k \neq i} p_{i k}^{l}(t)\right] w_{i j}^{l}(t) .
$$

Here, the time-dependent quantity $p_{i j}^{l}(t)$ is defined as

$$
p_{i j}^{l}(t)=\frac{1}{T}\left|\int_{t-T}^{t} e^{i\left[\phi_{i}^{l}\left(t^{\prime}\right)-\phi_{j}^{l}\left(t^{\prime}\right)\right]} d t^{\prime}\right| .
$$

Notice that $p_{i j}^{l}$ quantifies the average phase correlation between oscillators $i$ and $j$ of layer $l$ over a characteristic memory time $T$. Equation (2) yields the strengthening of the connections between those units, which are phase correlated across $T$, verging therefore the well-known Hebbian learning process [24].

From the solutions of Eqs. (1) and (2), the microscopic signal of each unit in each layer can be estimated at all times as $x_{i}^{l}(t)=\sin \left[\phi_{i}^{l}(t)\right]$, while the macroscopic signal of each layer can be expressed as $X_{l}(t)=\sum_{i=1}^{N} x_{i}^{l}(t)$. The spectral properties are analyzed by the wavelet transform [25], a well-known tool suitable for analysis of various nonstationary processes (as it is the present case, where signals come from layers whose structure of connections evolves actually in time).

As an initial, descriptive example, we start with illustrating the case of a monolayer network $\left[M=1\right.$ and $\lambda_{2}=0$ in Eq. (1)], for an intralayer coupling value $\lambda_{1}=0.5$, and for $T=100 \mathrm{~s}$. This parameter corresponds to the partial synchronization in the model network and the emergence of structural clusters in its topology [18].

To examine the dynamics $X(t)$ in both the time and frequency domains, the wavelet power spectrum can be calculated as $W(f, t)=|M(f, t)|^{2}$, where $M(f, t)$ is the complex wavelet surface defined with the aid of continuous wavelet

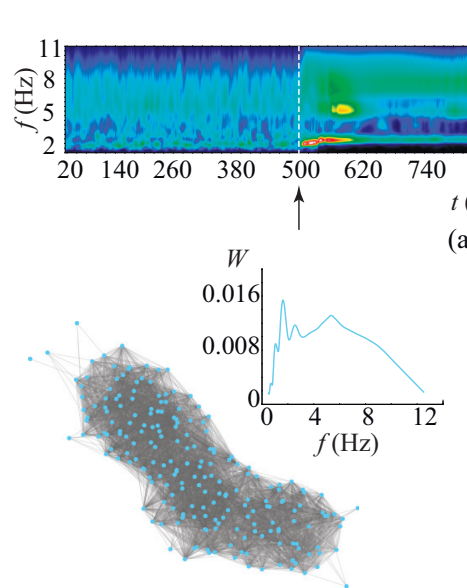

(b)

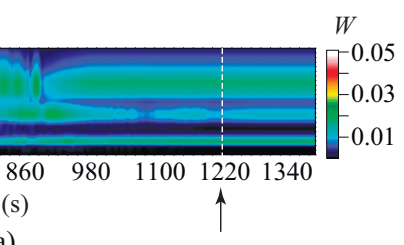

(a)

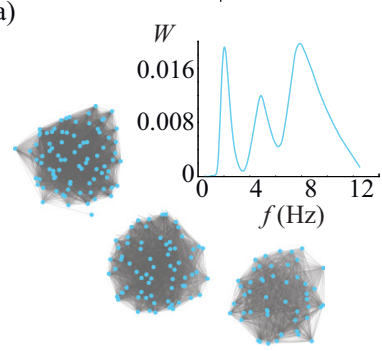

(c)
FIG. 1. (a) Wavelet decomposition of the macroscopic signal, produced by a monolayer network of phase oscillators under the effect of adaptive mechanism. Lower row: momentum distributions of the wavelet energy and the network topologies. Panel (b) refers to $t_{1}=500 \mathrm{~s}$ (i.e., before adaptation starts to take place in the system), while panel (c) is obtained for $t_{2}=500 \mathrm{~s}$ (i.e., after the adaptive process has produced a time independent network's topology). Both instants at which the lower panels are obtained, are indicated with vertical arrows in panel (a). $\lambda_{1}=0.5, T=100 \mathrm{~s}$. See main text for all other specifications.

transform $[25,26]$,

$$
M\left(f, t^{\prime}\right)=\sqrt{f} \int_{-\infty}^{+\infty} X(t) \psi^{*}\left[\left(t-t^{\prime}\right) f\right] d t
$$

(the symbol "*" denotes the complex conjugation), with the Morlet mother wavelet,

$$
\psi(\zeta)=\frac{1}{\sqrt[4]{\pi}} \exp (j 2 \pi \zeta) \exp \left(-\frac{\zeta^{2}}{2}\right)
$$

Figure 1(a) reports the evolution of the wavelet power spectrum $W(f, t)$ of the macroscopic signal $X(t)$. In the absence of the adaptive mechanisms $\left(t<t^{A}\right)$, the wavelet energy is distributed almost homogeneously over all range of frequencies [see the plot $W(f)$ in Fig. 1(b)], as well as the network is a single-component graph characterized by a highly homogeneous distribution in the link strengths. As $t$ exceeds $t^{A}$, the network structure evolves: the links between synchronized elements are strengthened, while the weakly synchronized nodes progressively loose their connections. After transient processes have expired, this leads to the appearance of three well-structured clusters, within which the elements exhibit frequency synchronization [see Fig. 1(c)]. The stationary momentum wavelet spectrum exhibits, therefore, three isolated peaks (corresponding to the spectral components of the signals taken from the three different clusters), and the structure of the layer becomes modular [with the three frequency clusters forming three densely connected clusters of nodes, as schematically illustrated in the lower plot of Fig. 1(c)].

A much richer scenario characterizes the case of a twolayered network [15-19], which is schematically illustrated in Fig. 2(a), and corresponds to setting $M=2, \lambda_{2}=0.005$, 


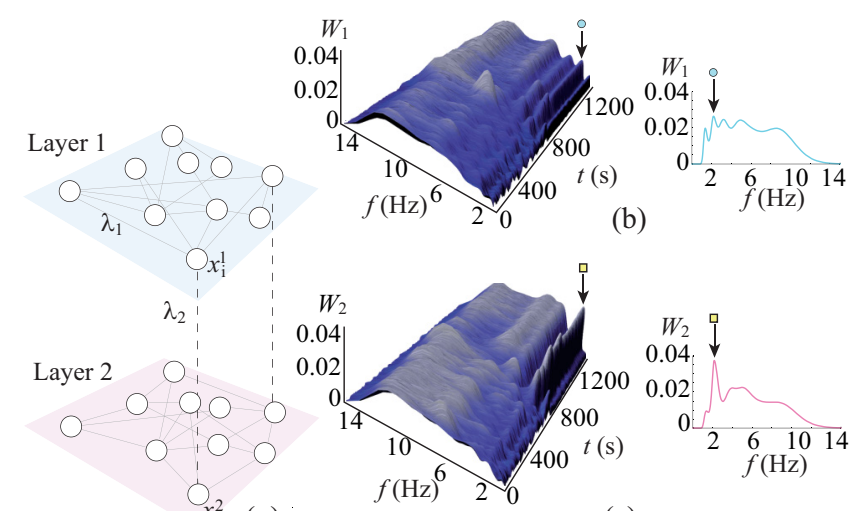

(a)

(c)

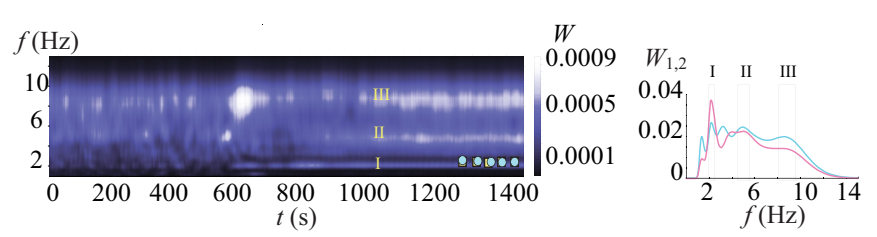

(d)

FIG. 2. (a) Schematic illustration of the two-layered network. Top right panels: $W_{1}(f, t)$ [panel (b)] and $W_{2}(f, t)$ [panel (c)] (see text for definitions), and the momentum distributions of the wavelet energy $W_{1}\left(f, t^{*}\right)$ [blue curve in panel (b)] and $W_{2}\left(f, t^{*}\right)$ [red curve in panel (c)], obtained for $t^{*}=1400 \mathrm{~s}$. (d) The surface of wavelet product Eq. (6). $\lambda_{1}=0.5, \lambda_{2}=0.005$, $T=100 \mathrm{~s}$. See main text for all other specifications.

$\lambda_{1}=0.5$ in Eq. (1). Here, the units have two types of connections: one set of links accounting for intralayer interactions (i.e., those befalling among elements of the same layer) and another set of links (the interlayer links) determining the coupling between elements of different layers. Under the simultaneous effect of intra- and interlayer couplings, the elements belonging to different layers, group into either identical and synchronous clusters (for relatively large values of $\lambda_{2}$ ) or distinct and asynchronous clusters (for small values of $\lambda_{2}$ ). The used set of parameters corresponding to partial synchronization between the layers implies the presence of identical clusters along with nonidentical ones. The macroscopic signals produced by the two layers are $X_{1,2}(t)=\sum_{i}^{N} \sin \left[\phi_{i}^{1,2}(t)\right]$.

The upper right part of Fig. 2 reports the wavelet energy distributions $W_{1,2}(f, t)$ for $X_{1}$ [Fig. 2(b)] and $X_{2}$ [Fig. 2(c)]. As in the previous case, one clearly sees that the wavelet energy is uniformly distributed for $t<t^{A}$ in both layers. For $t>t^{A}$, the spectral properties of each layer become similar to those of the single-layer case (the adaptive process within each layer leads to the formation of clusters and thus to a fragmentation of the wavelet spectrum), but, due to the initial mismatch in the inter-layer topology, the spectra of the two layers are different [compare the momentum distributions $W_{1,2}\left(f, t^{*}\right)$, for $t^{*}=1,400 \mathrm{~s}$, reported at the right of Figs. 2(b) and 2(c)].

While fragmentation of the wavelet spectra is caused by local synchronization (affecting independently the oscillators within each layer), several frequency ranges exhibit a local increase of the wavelet energy in both layers. The latter is associated with the emergence of global synchronization and is a consequence of the action of intralayer connections. Such
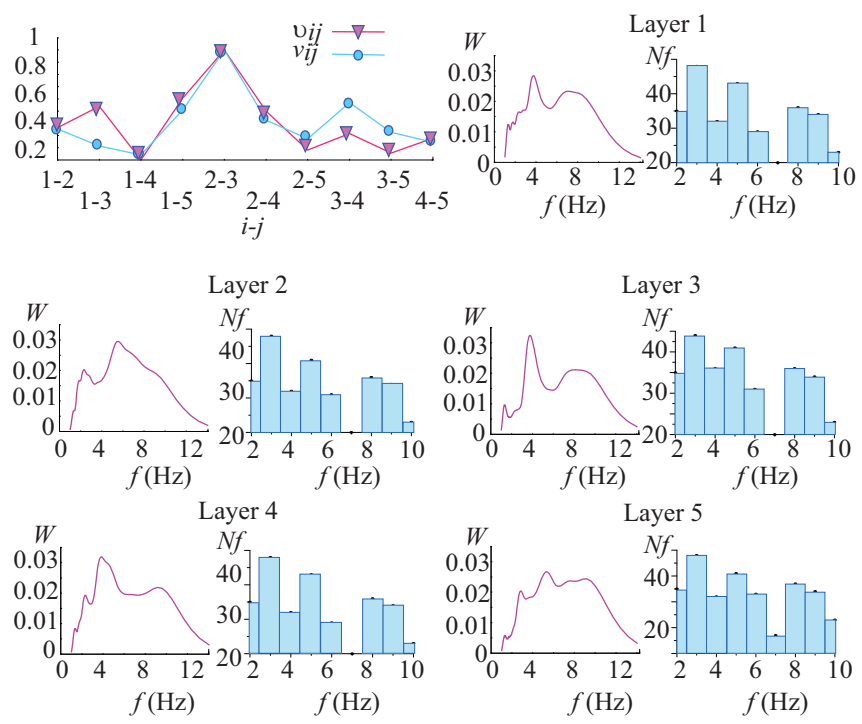

FIG. 3. Five-layered network $(M=5)$ for $\lambda_{1}=0.5, \lambda_{2}=0.005$, $T=100 \mathrm{~s}$. The upper left panel reports the two similarity functions of Eqs. (7) and (8) (see text for definitions). All other panels report the wavelet energy spectra $W(f)$ (left plots) and the effective frequency distributions $N(f)$ (right histograms) for the different layers, calculated at $t^{*}=1500 \mathrm{~s}$.

frequency bands are easily localized on the surface of the wavelet spectra product,

$$
W(f, t)=W_{1}(f, t) \times W_{2}(f, t) .
$$

In Fig. 2(d), the bands where local maxima of $W(f, t)$ are observed are marked as I, II, and III. In particular, the frequency band I is characterized by a significant increase of the wavelet energy in both layers. Inside band I, the frequency value where the wavelet energy of the first (second) layer reaches its maximum is marked by a solid circle (square) in Fig. 2(b) [2(c)]. The right plot of Fig. 2(d) discloses that the locations of the local maxima of $W_{1}$ and $W_{2}$ inside region I match perfectly, which, in turn, is a proof of synchronization between the layers.

Next, we consider the case of a five-layered network $(M=5)$, with inter- and intralayer links arranged in a way similar to the two-layer case. The degree of similarity $v_{i, j}$ between layers $i$ and $j$ can be defined (at a macroscopic scale) as the integral of the difference between the corresponding wavelet energy distributions $\hat{W}_{i, j}\left(f, t^{*}\right)$ taken at $t^{*}=1400 \mathrm{~s}$ (i.e., at the moment at which a stationary state has emerged, and the network topology does not change in time, except for small, residual, fluctuations affecting the intralayer links $w_{i, j}$ ):

$$
v_{i, j}=\left[\int_{f_{1}}^{f_{2}}\left|\hat{W}_{i}(f)-\hat{W}_{j}(f)\right| d f\right]^{-1},
$$

where $f_{1}=2 \mathrm{~Hz}$ and $f_{2}=10 \mathrm{~Hz}$ are the bounds of the frequency range for which global synchronization is analyzed. The value $\hat{W}(f, t)=\left(f_{2}-f_{1}\right) W(f, t) / \int_{f_{1}}^{f_{2}} W\left(f^{\prime}, t\right) d f^{\prime}$ for each frequency $f$ quantitatively characterizes the contribution of the corresponded spectral component in the whole wavelet power spectrum of the considered macroscopic signal.

The obtained values are shown in Fig. 3 (upper left panel, with solid blue circles). One easily sees that, due to the 
interplay between the adaptive evolution of links within each layer and the interlayer interactions (which may differ for different pairs of layers), the degree of similarity $v_{i, j}$ is distributed inhomogeneously [i.e., without an apparent order with respect to the pairs $(i . j)$ of layers].

Likewise, one can define another degree of similarity $v_{i, j}$ (at a microscopic scale, i.e., based on the states of the individual oscillators) as

$$
v_{i, j}=\left[\int_{f_{1}}^{f_{2}}\left|N_{f_{i}}(f)-N_{f_{j}}(f)\right| d f\right]^{-1},
$$

where now $N_{f_{i}}(f)$ is the effective frequency distribution of the oscillators in the $i$ th layer (i.e., the conveniently normalized number of oscillators of the $i$ th layer displaying an effective frequency $f$ ). The values of $v_{i, j}$ reflect then the similarity in terms of the number of oscillators (belonging to the $i$ th and $j$ th layers) exhibiting synchronous oscillatory behavior at the selected frequency, or equivalently, the similarity of structural clusters formed within the two layers. The upper left panel of Fig. 3 reports also (with solid pink triangles) the values of $v_{i, j}$ for each pair of layers and immediately beholds the remarkably good correspondence between the two measures, Eqs. (7) and (8).

Our examples demonstrate that homophilic and homeostatic adaptive principles ordain the structure and dynamics of multilayer networks into states where local and global synchronization (within specific frequency bands) can be revealed from measurements of microscopic and macroscopic quantities. The results, obtained for the considered model of multilayer network, demonstrate that the proposed approach, based on continuous wavelet transform, allows to effectively describe the dynamics of the complex networks on microscopic and macroscopic scale. It is important to know that the proposed method is based on the relation between the values of the spectral energy, calculated across wide bands frequency by means of continuous wavelet transform, and do not use directly the instantaneous phases of the signals. This means that the proposed approach is useful for the analysis of complex nonstationary signals, which do not have the well-pronounced component in the spectrum, e.g., EEG signals. Such conclusion is of relevance for the experimental study of neural networks in the brain, where spectral properties are actually related to the various forms of sleep-wake and cognitive activity.

\section{NEUROPHYSIOLOGICAL DATA}

At a physiological level, available recordings (through EEG or MEG) provide typical samples of brain dynamics at its macroscopic level. They reflect, indeed, integrated extra-cellular voltage changes of neural ensembles, located in the vicinity of the recording electrode. In parallel (and with the aid of intra- or extracellular single unit recordings), an operator is endowed with the opportunity of browsing the activity of a single neuron, inspecting brain dynamics at its microscopic level.

In the current study, local field potential (LFP) and single unit recordings were obtained in three-month-old genetic absence epilepsy rats (GAERS), a very similar genetic absence model as the earlier mentioned WAG/Rij model, under
Fentanyl-Droperidol anesthesia. A $1 \mathrm{M} \Omega$ Tungsten electrode was lowed in the deep layers of the somatosensory cortex, and another $1 \mathrm{M} \Omega$ Tungsten electrode was placed into the posterior thalamic nucleus. LFP and unit recordings of a given brain structure were gathered by the same electrode. Band-pass filters between $500 \mathrm{~Hz}$ and $10 \mathrm{kHz}$ were applied for the unit recordings, digitalized at a constant sampling rate of $20 \mathrm{kHz}$ by the SPIKE2 recording software [27,28]. LFP signals were filtered between 1 and $100 \mathrm{~Hz}$ and digitalized at a constant sampling rate of $1 \mathrm{KHz}$. All experimental procedures were performed in accordance with the guidelines of the council of the European Union of the 24th November 1986 (86/609/EEC), which were approved by local authorities (review board institution: LandesamtfürNatur, Umwelt und Verbraucherschutz Nordrhein-Westfalen; approval ID No.: 87-51.04.2010.A322).

Figure 4(a) illustrates the typical setup under which measurements are performed. In Fig. 4(b), we report the record of the EEG signal, as well as that of its underlying microscopic components (the activity of one or a few neurons) measured by the same electrode as the EEG signal. Since also the neuronal spiking is recorded with an extracellular electrode, it should be noted that it cannot be guaranteed that the spiking activity represents a single neuron. Therefore, spike sorting, using principle component analysis, was applied prior to analysis. Macroscopic and microscopic signals are acquired in two different (yet reciprocally connected) brain structures: cortex (S1) and thalamus (PO), which enables us to give ground to the above discussion about interrelationships between local and global synchronization processes.

In Fig. 4(b), we report the record of the EEG signal, as well as that of its underlying microscopic components (the activity of one or a few neurons) measured by the same electrode as the EEG signal. Since also the neuronal spiking is recorded with an extracellular electrode it should be noted that it cannot be guaranteed that the spiking activity represents a single neuron. Therefore, spike sorting, using principle component analysis, was applied prior to analysis.

In the recordings, a transition is observed from normal, physiological brain activity of the rat toward a pathological, hypersynchronous behavior, corresponding to the occurrence of an epileptic seizure (the so-called spike-wave discharge (SWD) due to the specific waveform of the electroencephalographic signals with well-pronounced large amplitude sharp peaks and slow waves [29,30]) and involving an abiding change in local and global synchronization properties of the brain network.

Before seizure starts, the registered macroscopic activities of cortex and thalamus are complex signals with continuous power spectra (see Figs. 4(c)-4(e) for $t<8.4 \mathrm{~s}$ ). Such a behavior actually corresponds to cells firing spontaneously and in a uncorrelated manner. At the onset of the seizure, instead, cells of cortex and thalamus start to exhibit a correlated bursting activity, which gives rise to the regularly repeated spike pattern (followed by a wave pattern) that is seen in the macroscopic recordings (Fig. 4(b) for $t>8.4 \mathrm{~s}$ ). A similar increase in phasic coupling during SWD can be seen for intracellular recordings of absence epileptic rats in VB and RTN neurons [31]. 


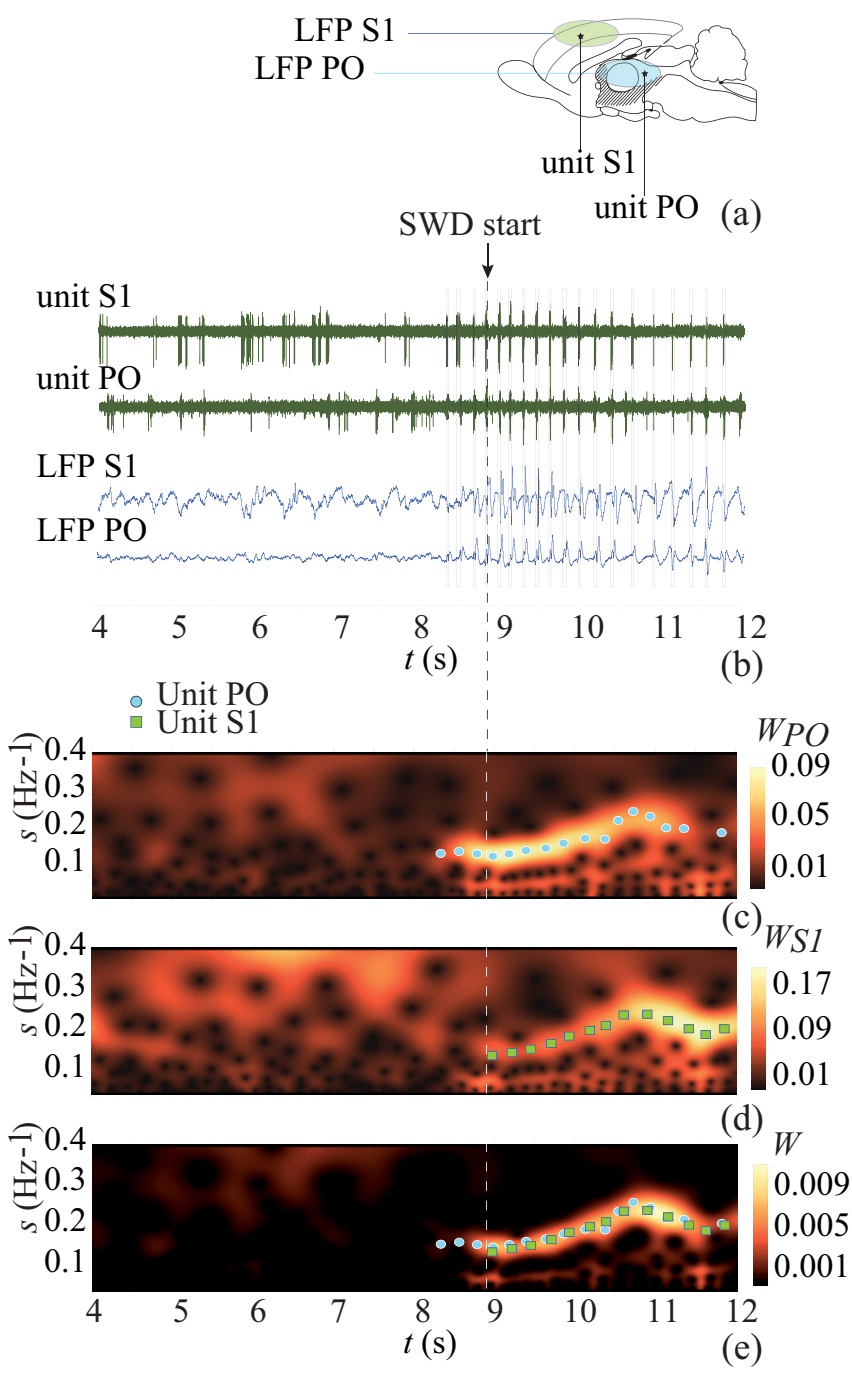

FIG. 4. (a) Schematic illustration of the experimental setup. The panel is a drawing of the rat's brain, with marked regions of cortex and thalamus, which contain the location of the recording electrodes that actually register the group neuronal activity by means of local-field potential (LFP) and the activity of the single cells (unit recordings). (b) The set of registered neurophysiological signals, reflecting the activity of single cells (unit S1 and unit PO) and the group activity in cortex and thalamus (LFP S1 and LFP PO). (c-e) Wavelet decompositions of the macroscopic signals. (c) $W_{\mathrm{PO}}(s, t)$ obtained from signal LFP PO, (d) $W_{\mathrm{S} 1}(s, t)$ obtained from signal LFP S1, and (e) $W(s, t)=W_{\mathrm{S} 1}(s, t) \times W_{\mathrm{PO}}(s, t)$. The solid circles and the solid squares shows the main spectral component of the signals, taken from the single cell in thalamus (unit PO) and cortex (unit S1), respectively. $s=1 / f$ indicates the timescale, and $f$ the linear frequency. The instant of time at which there is the onset of SWD is shown by an arrow and marked by a vertical white dashed line.

Like in the case of the network model, the distributions of wavelet energy $W_{\mathrm{S} 1}(s, t)$ and $W_{\mathrm{PO}}(s, t), s=1 / f$ (obtained from the macroscopic signals S1 and PO) change from an almost homogeneous configuration $(t \leqslant 8.4 \mathrm{~s})$ to a shape that is characterized by a local peak positioned in the frequency band corresponding to the epileptiform activity. According to the above discussion, this fact reveals that the onset of epileptic seizure establishes local synchronization within both cortex and thalamus, as well as global synchronization between these two regions of the brain. Indeed, by comparison of the surfaces $W_{\mathrm{S} 1}(s, t)$ and $W_{\mathrm{PO}}(s, t)$, and by consideration of the surface $W(s, t)=W_{\mathrm{S} 1}(s, t) \times W_{\mathrm{PO}}(s, t)$, one can see that the considered cells in the cortex and in the thalamus are synchronized at the frequency of the seizure and, moreover, they are synchronized with other cells belonging to the same part of the brain (see Fig. 4(e) for $t>8.4 \mathrm{~s}$ ).

Following the approach described in the previous section, one can analyze the dynamics of the thalamocortical network by means of a multichannel set of EEG recordings taken from Wistar Albino Glaxo from Rijswijk (WAG/Rij) rats [32]—a genetic animal model giving rise to absence epilepsy. In the experiment, six-month-old WAG/Rij rats were chronically implanted with stainless steel electrodes in layers 4 to 6 of the somatosensory cortex, as well as in (i) the posterior thalamic nucleus, (ii) the ventral-posteromedial thalamic nucleus, (iii) the anterior thalamic nucleus, and (iv) the reticular thalamic nucleus under deep isoflorane anaesthesia. Two weeks after surgery, EEG signals were recorded from theses structures in freely moving animals. Signals were filtered by a band pass filter with cutoff points at 1(HP) and 100(LP) and a $50 \mathrm{~Hz}$ Notch filter and digitalized by WINDAQ-recordingsystem (DATAQ-Instruments Inc., Akron, $\mathrm{OH}$ ) $[33,34]$ with a constant sample rate of $500 \mathrm{~Hz}$. Experiments were carried out in accordance with the Ethical Committee on Animal Experimentation of Radboud University Nijmegen (RU-DEC).

As a result, the recordings taken from three cortical and four thalamical electrodes are considered at different instants of time: (i) at the beginning of a seizure, (ii) at the end of a seizure, (iii) at a time at which the rat is in a state of active wakefulness, and (iv) at a time at which the rat is in a state of slow-wave sleep; refer to Fig. 6. Moreover (and according to the neurophysiological background of absence epilepsy [35]), the dynamics of the network is studied within three different frequency bands: (i) $\Delta_{f_{1}}$ (the low-frequency, LF, oscillation range $1-5 \mathrm{~Hz}$ ), (ii) $\Delta_{f_{2}}$ (the range $5-10 \mathrm{~Hz}$, SWD, of seizure activity), and (iii) $\Delta_{f_{3}}$ (the range of high-frequency, HF, sleep spindle range activity, 7-20 Hz). The wavelet energies $W_{\mathrm{LF}}, W_{\mathrm{SWD}}$, and $W_{\mathrm{HF}}$ are calculated as

$$
W_{\mathrm{LF}, \mathrm{SWD}, \mathrm{HF}}=\int_{t-\tau}^{t}\left[\int_{f \in \Delta_{f_{1,2,3}}} W_{i}(f, \xi) d f\right] d \xi,
$$

where $\tau=2.5 \mathrm{~s}$ is a time lapse chosen conveniently (and judiciously) to neglect spurious fluctuations, and $i$ is the number of the EEG channel.

The characteristic dynamical states of corticothalamocortical neuronal network of epileptic brain [the SWD (at the onset and the end), active wakefulness, and deep slow-wave sleep] are illustrated in Fig. 5, which reports typical EEG fragments recorded in cortex and thalamus, with the analyzed time epoches that are marked by rectangles and labeled as SWDO, SWDE, AW, and DSWS, respectively. Figure 6 (left panel) illustrates the ratio of the wavelet energies [Eq. (9)] calculated for all considered states (columns) and all channels (rows). One easily sees that during SWDO (i.e., when the epileptic seizure has just recently started), most of the wavelet energy (about 50\%) is concentrated in the range of SWD activity ( $W_{\mathrm{SWD}}$ ), both in the cortex and in the thalamus. This 


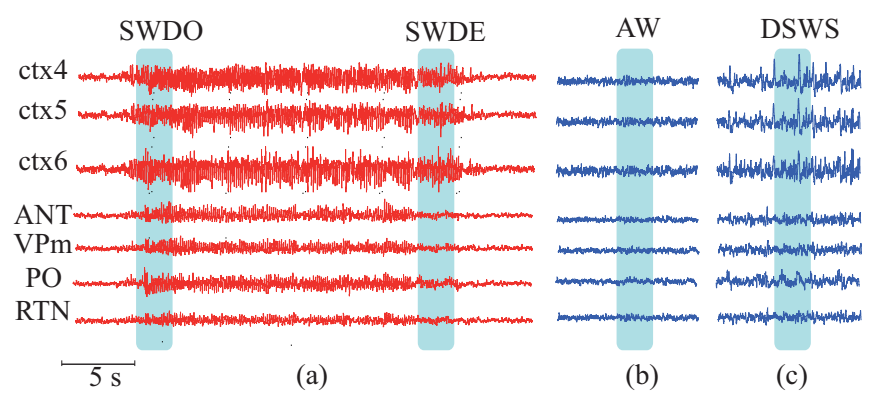

FIG. 5. Typical fragments of registered EEG signals, reflecting the activity of the neuronal group in cortex and thalamus during the epileptic discharge (SWD, a), during active wakefulness (AW, b), and during deep slow-wave sleep (DSWS, c). The epochs corresponding to the onset (SWDO) and end (SWDE) of SWD, to AW and DSWS are marked by rectangles.

reflects the fact that the synchronization level between neurons in the thalamocortical network of the brain increases at those frequencies which correspond to the band of the epileptic seizure $\Delta_{f_{2}}$. As the end of the seizure is approached (during SWDE), synchronization in the frequency band of the epileptic seizure $\Delta_{f_{2}}$ increases in the cortex, leading to an increase of $W_{\text {SWD }}$. At the same time, neurons in the thalamus start to go out of the hypersynchronous state, and the value of $W_{\mathrm{SWD}}$ decreases in the thalamus recordings.

During the normal physiological activity, i.e., active wakefulness (AW) state, the value of $W_{\mathrm{SWD}}$ is less in the cortex. At the same time, the wavelet energy increases in the frequency bands $\Delta_{f_{1}}$ and $\Delta_{f_{3}}$, both in the cortex and in the thalamus, which is caused by the neurons being involved in other types of brain activities (as, for instance, cognitive

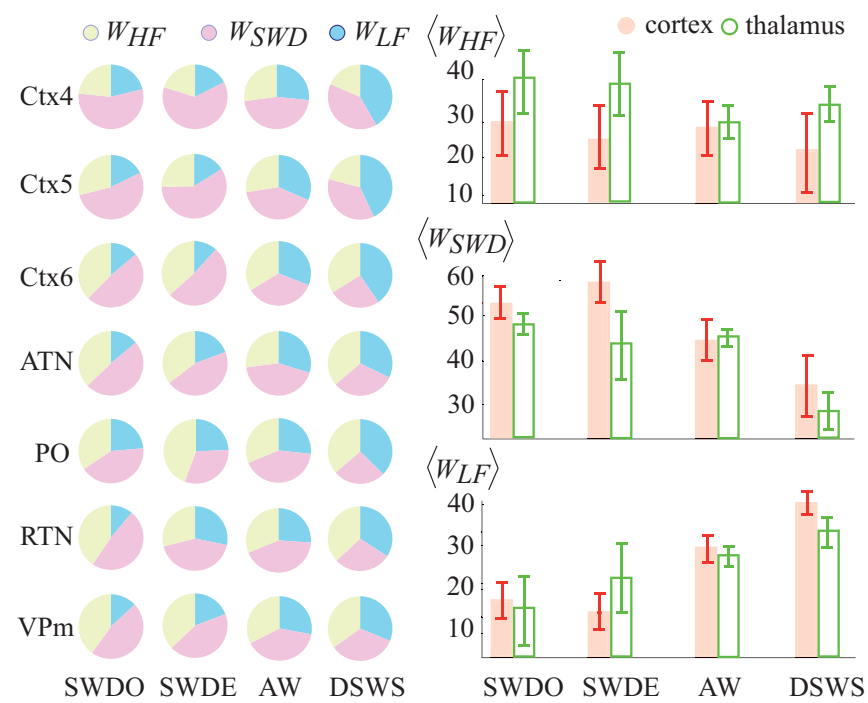

FIG. 6. Left panel: the ratio of the wavelet energies [Eq. (9)], calculated for all considered states (columns) and all channels (rows). Right panel: the energy values [Eq. (9)] averaged over the cortical (solid bars) and thalamical (empty bars) channels. The error bars stand for the maximal deviation within the channel set. Once again, SWDO, SWDE, AW, and DSWS stay for the SWD onset, the SWD end, the active wakefulness, and the deep slow-wave sleep, respectively. tasks). During deep slow-wave sleep (DSWS), most of the wavelet energy is concentrated in the low-frequency band $\Delta_{f_{3}}$, and $W_{\mathrm{LF}}$ experiences a significant growth, which actually corresponds to an increased number of the neurons producing low-frequency rhythms, and global synchronization between them both in cortex and in thalamus.

To quantitatively compare the changes in wavelet energies for the different states of the epileptic brain, we further report the values $\left\langle W_{\mathrm{LF}}\right\rangle,\left\langle W_{\mathrm{SWD}}\right\rangle$, and $\left\langle W_{\mathrm{HF}}\right\rangle$, which are the energy values [following Eq. (9)] averaged over the corticalthalamical recordings. These averaged values are shown in the right panel of Fig. 6, where solid and empty bars correspond to the cortex and thalamus recordings, respectively. The obtained results show that during the transition from the seizure onset to the seizure end (SWDO $\rightarrow$ SWDE) neurons in the cortex exhibit a growth of the synchronization level in the SWD frequency band $\Delta_{f_{2}}$, while the synchronization level in the thalamus decreases. As a consequence, the fact that the thalamic neurons abandon the synchronous state seems to be the cause of the epileptic discharge destruction.

At first glance this seems in contrast to previous observations of network dynamics preceding onset of SWDs [36]. These authors analyzed the spatiotemporal dynamics of interactions within and between widely distributed cortical sites using MEG from juvenile absence patients and reported for the $3 \mathrm{~Hz}$ band a preictal cortical long-range desynchronization $1.8 \mathrm{~s}$ before SWD onset, followed by local and long-range synchronization, all before SWD onset. A second MEG study focusing on preictal $\delta$ activity in children with childhood absence epilepsy, using nonlinear associations and time-frequency analyses of MEG signals noticed a preictal increase in power of the SWD band $(3 \mathrm{~Hz})$, an increase in local clustering, and a decrease in path length immediately preceding SWD onset, while during SWDs a general sharp increase in local connectivity and decrease in global connectivity was observed [37]. In a study toward cortical initiation and spreading of SWDs in the same genetic epilepsy model as was currently used, WAG/Rij rats, it was found that during SWDs cortical synchronization is increased as compared to the interictal periods [10], suggesting a common cortical phenomenon during SWDs in the rat model and in human patient patients. In WAG/Rij rats the dynamics of preictal widespread cortical activity were not studied in detail. Others [38-40] focused on corticothalamic, thalamocortical, and thalamothalamic interactions preictally and ictally in the model. The outcomes of their studies showed next to a general increase in coupling during the SWDs, also a complex dynamic pattern of interactions between different channel pairs during SWDs. Interestingly, preictal phase decoupling was seen between cortex and thalamus and between various thalamic sites [38] with pairwise phase consistency analyses, not with other methods, such as linear Granger causality [39]. Although this may or may not be related to the findings of Amor and Gupta cum suis, it is preferable to compare the human and rat data with the same analytical methods. Moreover, cortical spreading is undoubtedly different from corticothalamocortical spreading and or intrathalamic spreading. In all, it remains inconclusive whether in the WAG/Rij model preictal cortical desynchronization occurs comparable to the Amor et al. and Gupta et al. findings; this awaits experimental verification. A 
cortical grid covering the entire surface of the rats cortex might be necessary to obtain comparable rat data, notwithstanding the putative differences between human MEG and rat EEG data, the results obtained in juvenile absence patients versus WAG/Rij rats and the same analyses technique, the anatomical differences between humans and rats, and the location of the foci.

With the increase of $\left\langle W_{\mathrm{SWD}}\right\rangle$, the values of $\left\langle W_{\mathrm{LF}}\right\rangle$ and $\left\langle W_{\mathrm{HF}}\right\rangle$ decrease in the cortex, while the vice-versa occurs in the thalamus. $\left\langle W_{\mathrm{SWD}}\right\rangle$ decreases in the SWD frequency band with the growth of $\left\langle W_{\mathrm{LF}}\right\rangle$, whereas $\left\langle W_{\mathrm{HF}}\right\rangle$ remains practically unchanged. Such changes expose that the neuron groups in the cortex and thalamus interact with different intensities in the different frequency bands, depending on the specific brain state. For instance, synchronization in the SWD frequency band in the cortex becomes stronger at the end of the epileptic discharge. At the same time, in the thalamus one observes a decrease of synchronization in the SWD frequency band, and and increase in low- and high-frequency bands, i.e., the neurons start to interact more intensively producing the low- and highfrequency activity. During AW and DSWD, one observes a significant increase of the wavelet energy $\left\langle W_{\mathrm{LF}}\right\rangle$ with a simultaneous decrease of the energy, corresponding to the epileptiform pattern $\left\langle W_{\mathrm{SWD}}\right\rangle$ and alpha and theta activity $\left\langle W_{\mathrm{HF}}\right\rangle$.

A first conclusion that can be drawn is that neurons in the cortex and thalamus interact in the various frequency bands with different intensities depending on the specific state, leading to a variety of synchronous patterns. It should be noted that, along with the interaction between neurons located relatively close to each other in the cortex layers, the interaction between remote regions of the corticothalamocortical network, e.g., different nuclei of the thalamus, is also relevant for understanding processes leading to the hypersynchronous epileptic dynamics.

The interaction between cortical and thalamic neural activity preceding and during SWDs has been described in WAG/Rij rats [10] and in GAERS [41], another well validated and commonly used genetic absence model. Multisite cortical and thalamic field potential recordings showed increased coupling and synchronization between the cortical SWD initiation site and thalamus, while also synchronization, between cortical EEG and oscillatory activity in thalamus (ventral postero medial (VPM) and posterior) cells, gradually increased just prior to the cortical defined SWD and was maintained at an elevated value during the SWDs. Earlier, Pinault [42] described, also in GAERS, that action potentials in VPM and reticular thalamic nucleus occur in synchrony with the generalized SWDs.

As in the case of the multilayered network model, one can analyze the degree of the interaction between the different parts of the corticothalamocortical network during the generation of the different forms of activity. It can be assumed that in brain the neurons belonging to the different brain structures can be involved together in the generation of the certain rhythm. In this case, the wavelet spectra of the EEG signals, taken from these areas of brain, are expected to demonstrate the increase of the similarity. According to this, the strength of the interaction between the corresponded areas of brain can be estimated by the calculation of the degree of similarity $w_{i, j}$ via Eq. (7), where the limits of integration are chosen accordingly
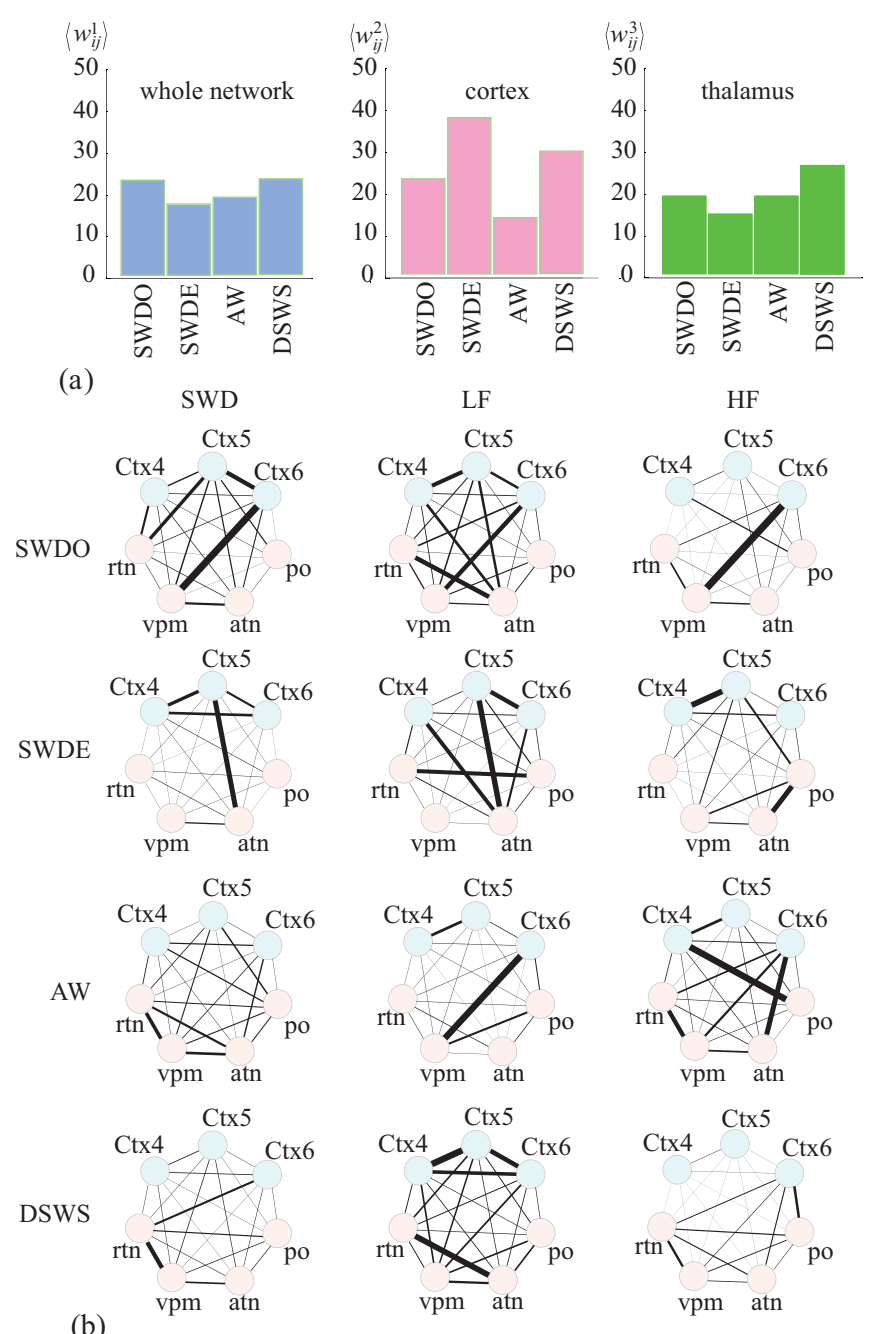

FIG. 7. (a) The mean value of the strength of interaction between the different parts of the thalamocortical neuronal network. The values [calculated for whole network $\left(w_{i j}^{1}\right)$, for the cortex $\left(w_{i j}^{2}\right)$, and for the thalamus $\left.\left(w_{i j}^{3}\right)\right]$ are averages of the coefficients $w_{i, j}$ over the considered parts of the brain. $w_{i, j}$ are estimated via Eq. (7), based on the similarity of the wavelet spectra. (b) The schematic illustration of the coefficients $w_{i, j}$, reflecting the degree of interaction between the different parts of the cortex and thalamus, for all considered states of brain network and frequency bands. The values of $w_{i, j}$ are shown by the increase (or decrease) of the line width, which connect the corresponded brain structures. Other stipulations are as described in the caption of Fig. 6.

to the frequency bands associated with the type of the rhythm (in our study the limits are defined by the bands $\Delta_{f_{1,2,3}}$ ).

Figure 7(a) reports the mean degree of the interaction between the areas of brain belonging to the cortex, the thalamus, and the whole neuronal network, calculated by averaging the coefficients $w_{i, j}^{k}$ over the corresponding region of the brain network (with $k=1$ for the whole corticothalamocortical network, $k=2$ for cortex and $k=3$ for thalamus). In Fig. 7(b), the values $w_{i, j}$ are shown by the increase (or decrease) of the line width, which connect the corresponding brain structures. From Fig. 7(a), one easily sees that the corticothalamocortical network is characterized by a high degree of global interaction 
at the onset of the seizure (SWDO state) and during the DSWS state, while the minimal value of $w_{i, j}^{1}$ is achieved for the SWDE state. Looking at the $w_{i, j}^{2}$ and $w_{i, j}^{3}$ values (corresponding to cortex and thalamus, respectively), one finds that the thalamical network exhibits a high level of global interaction during the SWDO and DSWD states, while during the SWDE state the different regions of thalamus interact weakly with each other. On the contrary, the cortical regions interact more strongly at the end of the epileptic seizure.

From Fig. 7(a), one can conclude that, along with the interaction within the different parts of cortex and thalamus, the increase of the interaction between these parts manifests itself as a key feature of the epileptic seizure. Indeed, at the beginning of the SWD (i.e., when the seizure occurs spontaneously) there is an increase not only within the cortex and thalamus separately, but also between them. At the end of seizure, despite the strong and stable cooperation between the parts of the cortex, the interaction between the thalamic nuclei and the cortical layers decreases. Based on our study it can be proposed that SWDs end due to the diminishment of the interaction between cortex and thalamus. Functional deactivation studies of the cortex and dorsal thalamus in WAG/Rij's and GAERS [43-45] demonstrate that an intact cortex and thalamus is a prerequisite for SWDs to occur. More fine-tuned experimental manipulations, for instance, with the aid of optogenetic techniques, are to be performed to clarify the causal role of particular interaction of specific neuronal groups in certain frequency bands in, e.g., seizure generation.

From Fig. 7(b), where the interaction in the corticothalamocortical network is shown for the different frequency bands, one can conclude that at seizure onset (the SWDO state), the different parts of neuronal network demonstrate a high level of interaction in the bands $\Delta_{f_{1}}$ and $\Delta_{f_{2}}$ (the low- and spike-wave oscillations frequency). The increase of the interaction in the $\Delta_{f_{1}}$ band is related to the presence of the low-frequency $\delta$ precursors, as shown in Ref. [46]. At the spike-wave discharge end (the SWDE state), there are still strong interactions in the cortex, related to the SWD-frequency band. At the same time, the parts of the thalamus interact in a much weaker way in this frequency band and, moreover, exhibit a significant decrease in the interaction with cortical neurons. When the seizure is finished and the animal exhibits active wakefulness, the different parts of corticothalamocortical network start to interact stronger in the frequency band $\Delta_{f_{3}}$, which corresponds to the generation of high-frequency brain rhythms. During the deep slow-wave sleep, such interactions can be observed in the band $\Delta_{f_{1}}$ of low frequencies, while in the other bands the different parts of the brain interact more weakly. During the AW state, the parts of the cortex and thalamus interact almost equally with each other, but the high degree of the interaction here is revealed in the high-frequencies range. Conclusion is that, during slow-wave sleep, the low-frequency oscillations ( $\delta$ waves) are generated by the neuron populations both in the cortex and in the thalamus, and this type of brain activity is characterized by a high degree of interlayer interaction in these parts of the brain as well as over the whole neuronal network of cortex and thalamus.

The degree of interaction between the cortical and thalamical EEG channels in the frequency band $5-10 \mathrm{~Hz}$ has

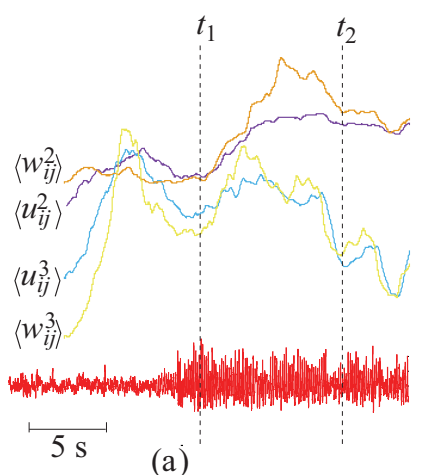

(a)

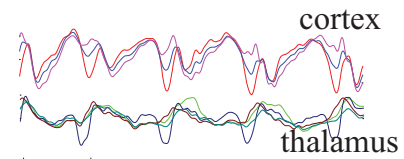

(b)

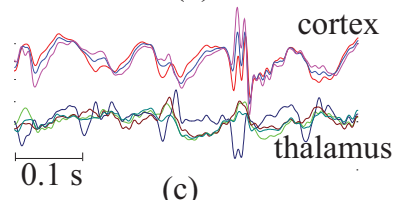

(c)
FIG. 8. (a) The mean values of the strength of interaction between the different parts of the thalamocortical neuronal network, calculated for the time interval corresponding to the development of the SWD. The values $w_{i j}^{2}$ (for the cortex) and $w_{i j}^{3}$ (for the thalamus) are averages of the coefficients $w_{i, j}$ over the considered parts of the brain. $w_{i, j}$ are estimated via Eq. (7), based on the similarity of the wavelet spectra. The values $u_{i j}^{2}$ (for the cortex) and $u_{i j}^{3}$ (for the thalamus) are also averages of the coefficients $u_{i, j}$ over the same parts of the brain, where $u_{i, j}$ are estimated based on the wavelet coherence [25]. The EEG samples, corresponded to the first seconds of the SWD development (b) (vicinity of time moment $t_{1}$ ) and (c) $t_{2}$.

also been considered on the time interval corresponding to the development of the SWD (see Fig. 8), using both the classical wavelet coherence and our approach. In Fig. 8(a), the wavelet coherence values [calculated for the cortex $\left(w_{i j}^{2}\right)$ and for the thalamus $\left.\left(w_{i j}^{3}\right)\right]$ are the averages of the coefficients $w_{i, j}$ over the considered parts of the brain, where $w_{i, j}$ are estimated via Eq. (7), based on the similarity of the wavelet spectra. The values [calculated for the cortex $\left(u_{i j}^{2}\right)$, and for the thalamus $\left.\left(u_{i j}^{3}\right)\right]$ are also the averages of the coefficients $u_{i, j}$ over the same parts of the brain, where $u_{i, j}$ are estimated based on the wavelet coherence [25]. One can see that the dependencies calculated with the different approaches behave similarly: there is an increase of the $w_{i j}^{2}$ and $u_{i j}^{2}$ values (cortex interactions) within the SWD region, and a decrease of the values $w_{i j}^{3}$ and $u_{i j}^{3}$ (thalamic interactions). In Figs. 8(b) and $8(\mathrm{c})$, the EEG samples are given for cortical and thalamical channels in the vicinity of the moment of the time $t_{1}$ chosen close to the SWD onset and in the vicinity of $t_{2}$ chosen close to the end of the SWD, respectively. One clearly sees that the oscillations in cortex and thalamus demonstrate phase synchronization. When comparing the cortical and thalamical EEG corresponding to the moments $t_{1}$ and $t_{2}$, it is possible to observe that, indeed, thalamic EEG start to desynchronize for $t=t_{2}$, while cortex still demonstrate strong synchronization.

At the same time, despite the similarity of the wavelet coherence and the proposed approach, the latter has the major advantage in the analysis of the multichannel data. While the wavelet coherence allows us to estimate the mutual coupling between the pair of signals, the proposed measure can be used for the detection of the global synchronous structures in the array of signals. It can be done by the multiplexing of the corresponded $\hat{W}_{i}(f, t)$ values in time-frequency domain [see Fig. 2(d)]. This feature of the proposed approach becomes 
important when processing the large amount of the multichannel data in real time and can be used in brain-computer interfaces or for the real-time monitoring of the human conditions.

\section{CONCLUSION}

Microscopic processes characterizing the interaction between the units of a network affect the properties of the network at any macroscopic scale. However, how this interdependency can be used in the reverse way (i.e., to reveal the nature of microscopic interactions by the study of the global changes of the network) is not generically obvious, especially when the dynamics of the single nodes is unknown. This is an important issue in neurophysiology, where data on the brain dynamics is available by means of electroencephalograms (EEG) or magnetoencephalograms (MEG), which actually measure the electric group activity of large ensembles of cells, while the state of individual neurons and the evolution of the links between them cannot be easily revealed. We have here demonstrated how to use the macroscopic properties of the network to get information on the network dynamics at a microscopic level.

Our analysis started with a model network of Kuramoto oscillators, for which the evolution of the links between the nodes is controlled by homophilic and homeostatic adaptive principles. We gave evidence that the increase of synchronization within groups of nodes (leading to the formation of structural synchronous clusters) causes also the defragmentation of the wavelet energy spectrum of the macroscopic signal. Considering a multilayer network model we revealed that nodes, belonging to different layers, interact with each other with different degrees of intensity. Namely, a strong interaction between the layers reflects the appearance of structural clusters (with the same spectral properties) on the layers. The degree of similarity between pairs of layers can be estimated as the integral of the difference between the wavelet energy distributions, and they display inhomogeneities over the considered pairs of layers.

The same phenomena were observed in a neurophysiological system considered, namely, in the corticothalamocortical network of the brain of a genetically epileptic rat [47], where the group electrical activity are registered by means of multichannel EEG. We demonstrated the possibility to determine the degree of interaction between the interconnected regions of the brain. Specifically, depending on the type of brain activity, we found that the neurons in cortex and thalamus interact in the different frequency bands with different degrees of intensity, which, in turn, leads to the formation of different synchronous patterns. Along with the interaction between neurons located relatively close to each other in the cortex layers, we gave evidence of interaction between more remote regions of corticothalamocortical network, e.g., different thalamic nuclei.
In addition, we detected strong synchronization of the cortical layers at the end of the epileptic seizures together with a decrease in their synchrony with thalamic nuclei. This is an indication of the attempt of the cortex, the location of the epileptic onset zone in this epilepsy model, to keep the seizure ongoing, which is corrupted by the thalamus. Interestingly, network analyses of the multichannel EEG in the same epilepsy model showed a high coherence and phase consistency between cortex and thalamus and between cortical layers during the seizure $[38,48]$ in agreement to what is well known in absence epileptic patients. A recent proposed scenario on how absence seizures spontaneously end, based on different types of advanced signal analyses, also mentioned that intrathalamic processes heavily contribute to the spontaneous ending of the seizure [39].

Our approach constitutes a practical technique for the investigation of brain neuronal network interactions, with the potential of getting a glance at interactions at a microscopic level by the analysis of the macroscopic signals commonly acquired in neuroscience studies. In particular, our study may be applied in a wide range of neurophysiological studies, which investigate functional brain network conductivities by means of EEG and MEG data during different forms of cognitive and behavioural tasks as well as for the study of pathophysiological brain processes.

Here we describe a model showing that local synchronization is the basis for long-range synchronization. The data, as obtained in epileptic rats, are just an example. Our model does not only illustrate how SWDs are generated within the corticothalamocortical network, but is also applicable to the broader topic of information processing within brain networks. The thalamocortical system is well described anatomically and functionally, this is mainly the case for the relay nuclei and the subgranual layers. However, and in contrast, the function and mode of information processing of higher-order thalamic nuclei is less well known [49]. In addition, the recognition and more detailed analysis of the immense diversity of, e.g., phase relationships and its implications for functional information processing within cognitive subdisciplines (e.g., attention or motor performances) is currently debated (see, for example, Ref. [50]). Maris et al. [51] proposed that diversity in phase relationships may support effective neuronal communication by enhancing selectivity and flexibility. Our findings support their general idea and demonstrate that dynamics and diversity are certainly present in pathophysiology leading to the occurrence of SWDs. It cannot be excluded that these principles also govern other corticothalamocortical processes, such as $\delta$ sleep and sleep spindles, next to cognitive functions.

\section{ACKNOWLEDGMENT}

The work has been supported by the Russian Science Foundation (Grant No. 16-12-10100).
[1] R. F. Betzel, S. Gu, J. D. Medaglia, F. Pasqualetti, and D. S. Bassett, Sci. Rep. 6, 30770 (2016).

[2] A. M. Hermundstad, D. S. Bassett, K. S. Brown, E. M. Aminoff, D. Clewett, S. Freeman, A. Frithsen, A. Johnson, C. M. Tipper,
M. B. Miller, S. T. Grafton, and J. M. Carlson, Proc. Natl. Acad. Sci. USA 110, 6169 (2013).

[3] S. Atasoy, I. Donnelly, and J. Pearson, Nat. Commun. 7, 10340 (2016). 
[4] S. Boccaletti, V. Latora, Y. Moreno, M. Chavez, and D.-U. Hwang, Phys. Rep. 424, 175 (2006).

[5] G. Buzsáki, Neuroscience 31, 551 (1989).

[6] C. Haenschel, D. J. Vernon, P. Dwivedi, J. H. Gruzelier, and T. Baldeweg, J. Neurosci. 25, 10494 (2005).

[7] R. M. Cichy, A. Khosla, D. Pantazis, A. Torralba, and A. Oliva, Sci. Rep. 6, 27755 (2016).

[8] T. J. Palmeri and I. Gauthier, Nat. Rev. Neurosci. 5, 291 (2004).

[9] A. Cavanna and F. Monaco, Nat. Rev. Neurol. 5, 267 (2009).

[10] H. K. Meeren, J. P. Pijn, E. L. Van Luijtelaar, A. M. Coenen, and F. H. Lopes da Silva, J. Neurosci. 22, 1480 (2002).

[11] M. Jalili, Sci. Rep. 6, 29780 (2016).

[12] E. M. Maynard, N. G. Hatsopoulos, C. L. Ojakangas, B. D. Acuna, J. N. Sanes, R. A. Normann, and J. P. Donoghue, J. Neurosci. 19, 8083 (1999).

[13] V. I. Nekorkin, Phys. Usp. 51, 295 (2008).

[14] S. Boccaletti, G. Bianconi, R. Criado, C. I. del Genio, J. GómezGardeñes, M. Romance, I. Sendiña-Nadal, Z. Wang, and M. Zanin, Phys. Rep. 544, 1 (2014).

[15] D. O. Hebb, The Organization of Behavior (Wiley, New York, 1949).

[16] M. McPherson, L. Smith-Lovin, and J. M. Cook, Annu. Rev. Sociol. 27, 415 (2001).

[17] R. I. M. Dunbar, J. Human Evo. 22, 469 (1992).

[18] S. Assenza, R. Gutierrez, J. Gomez-Gardenes, V. Latora, and S. Boccaletti, Sci. Rep. 1, 99 (2011).

[19] V. V. Makarov, A. A. Koronovskii, V. A. Maksimenko, A. E. Hramov, O. I. Moskalenko, J. M. Buldu, and S. Boccaletti, Chaos Solitons Fractals 84, 23 (2016).

[20] Y. Kuramoto, Chemical Oscillations, Waves, and Turbulence (Springer-Verlag, New York, 1984).

[21] S. H. Strogatz, Physica D 143, 1 (2000).

[22] A. Pikovsky, M. Rosenblum, and J. Kurths, Synchronization-A Universal Concept in Nonlinear Sciences (Cambridge University Press, Cambridge, 2001).

[23] S. Boccaletti, J. Kurths, G. Osipov, D. L. Valladares, and C. S. Zhou, Phys. Rep. 366, 1 (2002).

[24] S. Song, K. D. Miller, and L. F. Abbott, Nat. Neurosci. 3, 919 (2000).

[25] A. N. Pavlov, A. E. Hramov, A. A. Koronovskii, E. Yu. Sitnikova, V. A. Makarov, and A. A. Ovchinnikov, Phys. Usp. 55, 845 (2012).

[26] A. Hramov, A. Koronovskii, V. Makarov, A. Pavlov, and E. Sitnikova, Wavelets in Neuroscience (Springer, Heidelberg, 2015).

[27] http://ced.co.uk/products/spkovin
[28] T. Seidenbecher, R. Staak, and H. C. Pape, Eur. J. Neurosci. 10, 1103 (1998).

[29] E. Sitnikova and E. L. van Luijtelaar, Epilepsy Res. 71, 159 (2006).

[30] E. Sitnikova, A. E. Hramov, A. A. Koronovskii, and E. L. van Luijtelaar, J. Neurosci. Meth. 180, 304 (2009).

[31] D. Pinault and T. O'Brien, Thalamus Relat. Syst. 3, 181 (2005).

[32] K. Sarkisova and G. van Luijtelaar, Prog. Neuro-Psychopharm. Biol. Psych. 35, 854 (2011).

[33] http://www.dataq.com/

[34] A. Lüttjohann and G. van Luijtelaar, Neurobiol Dis. 47, 49 (2012).

[35] J. R. Tenney, H. Fujiwara, P. S. Horn, J. Vannest, J. Xiang, T. A. Glauser, and D. F. Rose, Ann. Neurol. 76, 558 (2014).

[36] F. Amor, S. Baillet, V. Navarro, C. Adam, J. Martinerie, and M. Le Van Quyen, NeuroImage 45, 950 (2009).

[37] D. Gupta, P. Ossenblok, and G. van Luijtelaar, Med. Biol. Eng. Comput. 49, 555 (2011).

[38] A. Lüttjohann, J. M. Schoffelen, and G. van Luijtelaar, Exp. Neurol. 239, 235 (2013).

[39] A. Lüttjohann and G. van Luijtelaar, Front. Physiol. 6, 16 (2015).

[40] M. V. Sysoeva, A. Luttjohann, G. van Luijtelaar, and I. V. Sysoev, Neuroscience 314, 75 (2016).

[41] P.-O. Polack, I. Guillemain, E. Hu, C. Deransart, A. Depaulis, and S. Charpier, J. Neurosci. 27, 6590 (2007).

[42] D. Pinault, J. Physiol. 552, 881 (2003).

[43] F. Scicchitano, C. M. van Rijn, and G. van Luijtelaar, PLoS ONE 10, e0133594 (2015).

[44] H. K. Meeren, J. G. Veening, T. A. Möderscheim, A. M. Coenen, and G. van Luijtelaar, Exp. Neurol. 217, 25 (2009).

[45] P.-O. Polack, S. Mahon, M. Chavez, and S. Charpier, Cereb Cortex 19, 2078 (2009).

[46] G. van Luijtelaar, A. Hramov, E. Sitnikova, and A. Koronovskii, Clin. Neurophysiol. 122, 687 (2011).

[47] G. van Luijtelaar and E. Sitnikova, Neurosci. Biobehav. Rev. 30, 983 (2006).

[48] E. Sitnikova and G. van Luijtelaar, Epilepsy Res. 84, 159 (2009).

[49] R. A. Mease, A. Sumser, B. Sakmann, and A. Groh, Cereb Cortex 26, 3461 (2016).

[50] S. Llufriu, E. Martinez-Heras, E. Solana, N. Sola-Valls, M. Sepulveda, Y. Blanco, E. H. Martinez-Lapiscina, M. Andorra, P. Villoslada, A. Prats-Galino, and A. Saiza, NeuroImage Clin. 13, 288 (2017).

[51] E. Maris, P. Fries, and F. van Ede, Trends Neurosci. 39, 86 (2016). 